\title{
Philosophiques
}

\section{Maurice Rainville, L'expérience et l'expression, Essai sur la pensée de Merleau-Ponty, Bellarmin, 1988, 133 p.}

\section{Martin Gagnon}

Volume 16, numéro 2, automne 1989

URI : https://id.erudit.org/iderudit/027090ar

DOI : https://doi.org/10.7202/027090ar

Aller au sommaire du numéro

Éditeur(s)

Société de philosophie du Québec

ISSN

0316-2923 (imprimé)

1492-1391 (numérique)

Découvrir la revue

Citer ce compte rendu

Gagnon, M. (1989). Compte rendu de [Maurice Rainville, L'expérience et l'expression, Essai sur la pensée de Merleau-Ponty, Bellarmin, 1988, 133 p.]

Philosophiques, 16(2), 410-418. https://doi.org/10.7202/027090ar d'utilisation que vous pouvez consulter en ligne.

https://apropos.erudit.org/fr/usagers/politique-dutilisation/ 
MaURICE RaINVILle, L'expérience et l'expression. Essai sur la pensée de Merleau-Ponty, Bellarmin, 1988, 133 p.

\section{par Martin Gagnon}

Il n'est pas rare d'observer qu'entre les premiers et les derniers travaux de certains auteurs, des ruptures apparaissent qui témoignent, le plus souvent, d'une volonté de se rapprocher de l'expérience, de tenir compte de nouveaux aspects et de trouver le langage susceptible de traduire au mieux les 
efforts que déploient la pensée afin de manifester une plus grande rigueur au sein de la recherche. Pensons à Schelling, à Husserl, à Heidegger, à Wittgenstein et à d'autres pour qui la philosophie fut aventure, aventure impliquant qu'il fallait parfois s'arrêter (au nom de l'expérience) pour pouvoir faire le point sur le chemin accompli et mesurer la nouvelle distance à parcourir, les nouveaux chemins à emprunter, quitte à revenir sur ses pas et devoir tout reprendre à zéro. On parle alors de rupture, de discontinuité, d'abandon, de "tournant», etc.

Dans son essai consacré à la pensée de Merleau-Ponty, Maurice Rainville n'ignore pas que le chemin menant de la première période (La structure $d u$ comportement, La phénoménologie de la perception) à la dernière (Le visible et l'invisible) manifeste l'équivalent d'un "tournant" qui ne saurait s'exprimer ni dans les termes d'une pure rupture, ni dans ceux d'une continuité sans faille philosophiquement pertinente. Il s'agirait peut-être d'une rupture dans la continuité, d'une tension originelle entre un langage traditionnellement déterminé et son effort pour rendre compte d'une expérience dont les enjeux débordent les ressources de ce langage ; tension qu'il s'agirait donc de saisir à sa source avant même (ou plutôt que) de s'engager dans une étude comparative portant sur l'allure générale des premiers et des derniers essais : "En d'autres termes, nous voulons aller, en deçà du fait de la rupture et celui de la continuité, à la source même de cette rupture et de cette continuité. »(p. 10)

Cette source, c'est évidemment dans les premiers travaux de MerleauPonty que Rainville la cherchera, et c'est en prenant pour fil conducteur « la portée ontologique de l'expérience » qu'il entend la dégager. L'ouvrage de Rainville se divise en deux parties principales : la première est consacrée à la signification du retour à l'expérience dans la première pensée de MerleauPonty; la seconde porte sur l'expérience perceptive comme telle et sur son rapport avec le langage ou l'expression.

Le premier chapitre de la première partie s'attaque d'emblée à une tension majeure de la pensée de Merleau-Ponty, soit le primat de l'expérience et le statut de l'expression. À travers le paradigme de la perception, nous dit Rainville, c'est l'expérience entendue comme " opération par laquelle nous nous donnons originairement un monde » (p. 17) que Merleau-Ponty cherche à rejoindre. Tel est le premier sens du retour à l'expérience. Mais si la perception prévaut comme propédeutique privilégiée pour accéder à ce que nous expérimentons, le retour à l'expérience ne signifie pas pour autant la réduction de toute expérience à celle rencontrée dans la perception. C'est d'abord et avant tout «à l'être présent et vivant » qu'il s'agit de revenir, et il faut retenir qu'à l'égard de cette préoccupation première, la perception n'a que le statut d'exemple méthodologique. Une seconde caractéristique du retour à l'expérience est que ce retour ne semble pas pouvoir être séparé des critiques que Merleau-Ponty fait valoir contre les prétentions de la pensée objective: «Le retour à l'expérience signifie la remise en question de la conception de la raison (...) selon laquelle la source de toute intelligibilité se 
retrouve dans un ordre qui transcende l'expérience et s'impose en même temps à celle-ci.» (p. 18) En effet, une attention fidèle aux données de la perception conduit à mettre en relief la genèse contingente du sens au sein de l'expérience et à remettre en cause, entre autres conceptions, celle de l'idéalisme qui exige que le sens soit donné de façon nécessaire par une conscience qui transcende le flux inorganisé du sensible.

Mais sice retour à l'expérience nous confronte à la contingence du sens donné dans la perception individuelle, comment concevoir alors l'expression philosophique de ce que nous expérimentons dans un langage dont les concepts n'ont de portée qu'à condition de désingulariser notre expérience? La signification abstraite du langage doit-elle être conçue comme appartenant à l'expérience, ou celle-ci ne doit-elle pas plutôt signifier en vertu d'une intelligibilité ou d'une «lumière » qui ne doit rien au langage? Rainville relève ici une tension majeure de la première pensée de Merleau-Ponty. S'il est vrai que notre perception des choses est perspectiviste, à savoir que les choses nous sont chaque fois données par profils, il n'en demeure pas moins que pour expliquer comment il est possible de saisir l'unité d'une chose pardelà (ou en deçà) de la multiplicité des aspects par lesquels nous l'appréhendons, force est d'admettre que même si ce pouvoir ne relève pas de la perception comme telle, il la traverse et ne peut pas, de ce fait, en être séparé : «...comme la perception est un aspect de la dialectique humaine et que la dialectique humaine implique en son fond une prise de distance, une négation ou un dépassement par rapport à ce qui est donné, la perception humaine ellemême doit être traversée par ce pouvoir de dépassement et ne pas pouvoir se réduire à une vue perspective. » (p. 29). Ceci viendrait donc compromettre le moment d'une expérience autonome par rapport au langage et antérieure à elle. Mais ce n'est là qu'une première position du problème fort complexe de l'expérience et de l'expression, un déblayage préliminaire visant en quelque sorte à préparer le terrain pour une enquête plus poussée, comme nous le verrons dans les derniers chapitres.

Pour l'instant, il s'agit de tirer au clair les conséquences critiques de ce retour à l'expérience; telle est la tâche du deuxième chapitre.

Si Merleau-Ponty se représente le but principal de la philosophie comme un retour à l'expérience, c'est nécessairement parce qu'il estime que celle-ci fut manquée (ou "dépassée ») par une certaine attitude philosophique. Cette attitude, Merleau-Ponty lui donne le nom général de pensée objective; qu'elle se présente sous la forme du réalisme ou de l'intellectualisme, l'auteur de la Pbénoménologie de la perception dénie à la pensée objective la prétention d'être « la seule voie d'accès au réel et à la vérité » (p. 35). On peut noter à ce sujer que la critique menée par Merleau-Ponty est toujours interne, c'est-à-dire qu'elle se place sur le terrain de l'adversaire lui-même pour montrer à quelies apories il aboutit s'il tire rigoureusement toutes les conséquences de ces principes. C'est ce que Rainville nous montre en exposant de façon détaillée la critique que fait Merleau-Ponty du réalisme et de l'intellectualisme. Je n'ai pas l'intention d'entrer ici dans le détail de ces 
analyses, mais il me semble indispensable d'en rapporter les conclusions. D'abord, en ce qui concerne la thèse réaliste, le retour à l'expérience nous apprend « /qu' June pensée de survol, une pensée qui ne serait pas assujettie à un point de vue, est impossible." (p. 46) Pour ce qui est de la critique de l'idéalisme, Merleau-Ponty reconnaît bien à la méthode réflexive une valeur intrinsèque, mais il refuse cependant de suivre l'idéalisme lorsque celui-ci réduit la conscience à une pure pensée dont la tâche consiste à lier un divers empirique originellement dépourvu de sens. Une telle réduction manifeste en effet certains préjugés que Merleau-Ponty refuse et parmi lesquels on retrouve, pour ne parler que des principaux, la thèse de la sensation aveugle - thèse que l'idéalisme emprunte d'ailleurs à l'empirisme - et celle qui considère que le corps n'est «qu'une signification pure que la conscience a devant elle» (p. 53) et qui rend donc impossible toute idée de conscience incarnée et soumise au perspectivisme.

Le dernier chapitre de la première partie, intitulé « La portée philosophique du retour à l'expérience », cherche à préciser le sens véritable de la réduction phénoménologique dans la pensée de Merleau-Ponty: «Merleau-Ponty situe la critique de la pensée objective dans le jour de la réduction phénoménologique husserlienne. De quelle idée exacte de la réduction Merleau-Ponty rapprochet-il sa propre démarche? » (p. 55) La question est intéressante, et plus encore la réponse apportée par Rainville. Si la réduction phénoménologique signifie, chez Husserl, la suspension de l'attitude naturelle de la conscience, la position de la conscience pure comme donatrice de tout sens et son corrélat, c'est-à-dire l'existence du monde par et pour la conscience (faisant ainsi de l'existence absolue du monde une absurdité), alors il est clair que ce n'est pas à ce sens de la réduction que Merleau-Ponty se réfère dans ses propres travaux: «Les démarches Husserl et de Merleau-Ponty sont, en un sens, l'inverse l'une de l'autre. Alors que Husserl met en suspens la croyance en un monde inhérente à l'expérience naturelle (...) Merleau-Ponty s' installe effectivement dans la science (...) et, en en poussant jusqu'au bout les conséquences, il se voit ramené à l'expérience naturelle comme à la source de toute vérité. " (p. 51)

De la réduction husserlienne, Merleau-Ponty n'aura finalement retenu que le dessein formel. Comme le dira encore Rainville : « $\grave{A}$ la différence de Husserl, ce n'est pas à l'expérience que Merleau-Ponty enlève son autorité, mais à la pensée objective» (p. 59), pensée dont Husserl lui-même se réclame dans son intention de fournir à la science un fondement absolu à partir d'une conscience constituante sans laquelle rien n'aurait de sens. De ce fait, Husserl aussi tombe sous la critique de l'intellectualisme. C'est sa conception technique de la pensée qui fait désormais l'objet d'une «épochè » de la part de Merleau-Ponty. Pour ce dernier, nous l'avons vu, ni le réalisme, qui se donne le monde tout fait, ni l'idéalisme, qui se le donne tout à faire, ne sont en mesure de «surprendre le monde dans le moment même de sa naissance. » (p. 63) La véritable tâche de la réduction pour Merleau-Ponty ne consiste donc pas à viser le fantôme objectif d'une conscience transcendantale, mais à reconduire le philosophe à l'ambiguïté de cette expérience au sein de laquelle les choses apparaissent comme de véritables « en-soi-pour-nous », et 
qui à cause de cela manifeste en quoi le monde, bien qu'à notre mesure, conserve toutefois ce fond d'étrangeté qui défie sans cesse toute mesure. Ainsi, dira finalement Rainville, « la voie reste ouverte à la reconnaissance de la portée ontologique de l'expérience» (p. 69, je souligne), c'est-à-dire à la reconnaissance de ce versant du monde qui résiste à toute constitution subjective.

Jusqu'ici, il s'agissait pour Rainville de dégager les modalités et les conséquences philosophiques du retour à l'expérience préconisé par MerleauPonty. La seconde partie de l'ouvrage, répondant au programme de départ, cherchera maintenant de façon plus directe à cerner les tensions qui habitent l'ceuvre du philosophe: «Nous voulons en particulier mettre en évidence dans certe description [ de l'expérience perceptive ] ce qui nous semble être un dépassement de la philosophie de la conscience et qui reste pris pourtant dans le cadre de cette philosophie elle-même. » (p. 75) La tension principale que recèle cette dialectique entre la philosophie de la conscience et son autre se manifeste assez clairement dans le rapport qui s'établit entre la description psychologique de l'expérience - qui, comme le montrera Rainville, dépasse les schèmes traditionnels de la philosophie de la conscience - et la réflexion de second degré qui porte sur cette description et qui, en tant que réflexion de second degré, s'inscrit « dans une obédience au point de vue de la conscience. » (p. 75) Tension, donc, entre le plan ontologique et le plan phénoménologique que Rainville désire maintenant dégager en partant de la notion d'être au monde. L'introduction de cette notion aura pour effet de mettre l'accent sur la communion originelle du monde et de la subjectivité, communion qui bouleverse de ce fait le dualisme cartésien du monde comme chose et du sujet comme conscience pour ouvrir la voie à une nouvelle conception du sujet en tant que «subjectivité incarnée », laquelle partage avec le monde une «étoffe commune » et est donc orientée par lui dans les diverses modalités de sa situation. Si la conscience métaphysique y perd au change, le corps, en revanche, apparaît désormais comme le médiateur premier dans la dialectique qui s'instaure entre notre vie personnelle et le monde: "La vie personnelle est sous-tendue par une existence pré-personnelle ou anonyme qui se confond avec la vie de mon corps. » (p. 78)

«Si l'introduction de la notion d' "être au monde" est destinée à remplacer la dichotomie de l'être comme chose et de l'être comme conscience, elle fait néanmoins apparaître une nouvelle différence, mais cette fois à l'intérieur du rapport intentionnel au monde, entre l'existence anonyme et l'existence personnelle.» (p. 82) Compte tenu de la dimension ontologique introduite par la présence de la notion d'être au monde dans la première pensée de Merleau-Ponty, compte tenu surtout des bouleversements qu'entraîne cette notion à l'égard du schème traditionnel conscience/objet, Rainville est amené à réviser le jugement que Merleau-Ponty lui-même portait sur le cadre à l'intérieur duquel ses premières analyses s'étaient déroulées. Selon ce dernier en effet, la Phénoménologie de la perception demeurait tributaire de l'appareillage conceptuel fixé par le point de vue de la conscience. Or, Rainville montre au contraire "jusqu'à quel point la pensée du premier 
Merleau-Ponty s'éloignait elle-même de ce point de vue » (p. 87), ce qui suggère que s'il y a bel et bien des différences entre la Pbénoménologie de la perception et Le visible et l'invisible, ces différences s'expliquent moins par le passage brusque d'une philosophie de la conscience à une philosophie branchée sur l'Être que par la volonté d'exprimer l'unité fondamentale de la subjectivité et du monde dans un langage qui soit redevable de moins de choses aux catégories traditionnelles qu'il ne l'est dans la Pbénoménologie de la perception: "L'unité de l'intériorité et de l'étendue dans un seul être qui est mon corps ne trouvait pas beaucoup d'autres moyens de s'exprimer que des expressions qui respiraient le dualisme de l'ontologie classique (...) Dans Le visible et l'invisible, cette unité [ la même donc - M.G.] s'exprime par des métaphores - réversibilité, circularité, etc. » (p. 85)

La portée ontologique de la notion d'être au monde est la source, on le voit, d'une tension constante entre une pensée dont l'intention première est de coller aux données fondamentales de l'expérience et un langage qui, dans la mesure où il tend à respecter l'impératif épistémologique de réflexion sur l'expérience, ne parvient pas tout à fait à s'autonomiser de l'héritage classique.

C'est une tension semblable qu'exprime la notion de transcendance dans les premiers travaux de Merleau-Ponty et, chose étonnante, c'est encore une fois à cause de ce souci à l'endroit de la portée ontologique de l'expérience que cette tension apparaît.

Le chapitre 5, prenant pour thème la section de la Phénoménologie de la perception intitulée « La chose et le monde naturel », demande ce qu'il en est de la réalité de la chose comme telle, c'est-à-dire de la chose en tant qu'elle se présente comme "hostile et étrangère », comme l'autre de ce qui nous est donné dans notre expérience perceptive. Mais ceci pose problème car "cet examen d'ordre ontologique, s'il doit vraiment rejoindre la chose réelle, ne pourra que se lier à l'expérience..." (p. 92) Ceci revient à dire que, pour le philosophe dont la tâche consiste à revenir à l'expérience, il ne semble pas possible d'engager un discours sur l'être même des choses sans que cet être soit d'une certaine manière présenté à la subjectivité - même si cette présentation n'épuise pas la transcendance de l'être en question et qu'elle ne la manifeste que sous l'un de ses aspects possibles. Rainville remarque qu'il est en effet question de transcendance chez Merleau-Ponty : le monde et les choses «transcendent les perspectives que je prends sur eux. » (p. 92) Or, que faut-il entendre au juste par transcendance? Recourant à Heidegger pour répondre à cette délicate question, Rainville finit par établir que la transcendance désigne l'être du Dasein en tant que celui-ci s'ouvre au monde entendu comme totalité. «Le monde comme totalité n'est pas un existant, ce n'est pas non plus la somme des existants, mais ce qui détermine ceci ou cela comme comptant ou pouvant compter pour le Dasein comme existant." (p. 96) Tel est le sens de la transcendance, non seulement pour Heidegger, mais vraisemblablement aussi pour Merleau-Ponty, affirme Rainville. Thèse fascinante, mais difficile également. Le côté «en-soi» de la chose ne serait 
alors « qu'une des concrétions possibles que projette autour de lui mon être comme transcendance » (p. 97), ce qui permet à la transcendance, d'une part, de se réfléchir en l'une quelconque de ses modalités concrètes - il faut de fait qu'elle se manifeste en « un faire particulier " pour que le sujet philosophant en fasse l'expérience - mais aussi de se « réserver » en tant que transcendance, c'est-à-dire d'être toujours au-delà (qu'on me pardonne le pléonasme!) des « faire particuliers » qui la concrétisent sous l'un ou l'autre des aspects donnés dans la perception. Ainsi, comprendre comment la chose est en-soi revient à comprendre comment elle est en-soi-pour-nous : " Elle est pour nous en tant qu'elle est atteinte dans un acte de perception (...) et elle est en soi en tant qu'elle est une des concrétions possibles du monde-esquisse que projette mon être comme transcendance. » (p. 99) Les deux aspects sont liés, quoique distincts, et c'est la non-reconnaissance de cette différence dans l'unité qui explique, selon Rainville, l'étonnement de certains commentateurs devant des expressions comme "plénitude de l'être» qui, dans la mesure où elles renvoient à cette altérité foncière des choses et du monde, ne semblent pas compatibles avec cet univers phénoménalisé ou subjectivisé que MerleauPonty rencontre au niveau de ses descriptions psychologiques. L'ambiguité est évidemment renforcée par l'emploi de termes traditionnellement déterminés dans la Phénoménologie de la perception: «Qu'est-ce, par exemple, que la foi perceptive, que Merleau-Ponty désigne aussi par l'expression d' "opinion originaire", sinon mon être même comme transcendance projetant le monde?" (p. 103) Encore une fois, c'est la portée ontologique de l'expérience qui semble responsable des tensions majeures de la pensée de Merleau-Ponty, et par conséquent des reproches fondamentaux qu'on a pu adresser à cette pensée.

Le dernier chapitre intitulé «L'expérience et l'expression» se penche sur la notion de cogito tacite et sur son rapport au langage. Le problème est posé comme suit : comment concevoir qu'une subjectivité, présente à soi dans ses actes singuliers, puisse en même temps transcender ces différents actes pour saisir comme unité? La notion de cogito tacite renvoie à une "présence silencieuse à soi " et semble permettre du même coup à la subjectivité de se saisir comme telle lors d'expériences privilégiées. "Mais elle n'est alors qu'un "sentiment de soi", elle r'est pas la pensée de la pensée " (p. 109), car il est manifeste que tout retour réflexif sur soi ne saisirait pas le cogito tacite, mais seulement le concept de cogito, celui que Descartes met explicitement en ceuvre dans les Méditations. Or Rainville, à la suite de Merleau-Ponty lui-même, remarque que les conclusions du chapitre sur le Cogito ne sont pas reliées à celles que l'on retrouve dans le chapitre sur la parole, dans la Pbénoménologie de la perception: «Le Cogito tacite ne peut rendre compte que du sens perceptif (...) Mais il ne peut rendre compte de mon ouverture aux idéalités comme telles. " (p. 112) Le chapitre sur la parole rend impossible la définition du Cogito comme présence silencieuse à soi puisque la parole n'a pas pour but de traduire une pensée déjà auto-suffisante (quoique muette), mais bien de l'accomplir. La pensée du dernier MerleauPonty parle de la "naïveté d'un cogito silencieux", de son impossibilité. Il 
semble donc que l'auteur de la Phénoménologie de la perception rejoigne ici la tradition des Hegel, Heidegger et Blanchot pour qui « l'expression fait être un sens qui commence d'exister avec elle." (p. 116).

Mais d'un autre côté, Rainville tient compte du fait que Merleau-Ponty ne peut pas vouloir tout simplement sacrifier la thèse du cogito tacite à celle de l'expression puisque, pour parler comme Blanchot : "Le langage ne me donne une chose qu'en lui retirant sa réalité d'os et de chair». (p. 118) Substituer purement et simplement le symbole à la «chair du monde » n'est certainement pas ce que désire Merleau-Ponty en tant que phénoménologue. "Et pourtant, c'est par le langage seulement que l'expérience en arrive à prendre conscience d'elle-même.» (p. 119) Ainsi, la coexistence des deux thèses opposées dans la Pbénoménologie de la perception n'implique pas vraiment qu'il faille choisir l'une au détriment de l'autre, mais, qu'en maintenant la double-exigence de la présence silencieuse à soi et de l'expression de cette présence, le philosophe cherche toujours plus rigoureusement à amener l'expérience à l'expression de son propre sens. C'est donc dire qu'ultimement, c'est à l'expérience que le philosophe doit revenir puisque aussi bien, c'est toujours d'elle qu'il reçoit les tâches de sa pensée, et que c'est à elle que l'expression doit s'attacher si elle ne veut pas a priori lui imposer des schèmes et des concepts qui lui seraient étrangers.

« À la connaissance, dira Rainville en conclusion, Merleau-Ponty substitue l'expérience comme coexistence ou comme communion (...) Dès lors, l'ouverture au monde ne peut plus être le fait d'une pensée qui le survole, ma is le fait d'un sujet qui est situé en lui, qui en est.» (p. 126) Cette sensibilité à l'égard de "l'étoffe commune » du corps et du monde, à cette communauté ontologique qui les lie l'un à l'autre, explique les diverses tensions relevées dans l'ceuvre de Merleau-Ponty; tensions qui semblent toutes découler plus ou moins directement de celle, centrale, entre une pensée qui se veut fidèle à l'unité que révèle l'expérience, et un langage philosophiquement déterminé par les couples d'oppositions qui caractérisent la métaphysique: sujet/objet, perception/intellection, conscience/corps, etc. Langage que les premiers travaux ne pouvaient peut-être pas éviter et auquel Merleau-Ponty a sans doute essayé de faire dire plus qu'il ne pouvait.

Peut-on vraiment parler d'une philosophie de la conscience pour ce qui concerne les premiers travaux? Sartre notait déjà le tour "contre-traditionnaliste » de la pensée de Merleau-Ponty : «... c'est qu'il fait appel à la raison afin, justement, de démontrer par son moyen, les limites de la raison. » (cit. p. 35) N'est-ce pas la déconstruction au sens où l'entend, par exemple, Derrida? «Les mouvements de déconstruction ne sollicitent pas les structures du dehors. Ils ne sont possibles et efficaces, ils n'ajustent leurs coups qu'en habitant ces structures (...) empruntant ì la structure ancienne toutes les ressources stratégiques de la subversion (...) l'entreprise de déconstruction est toujours d'une certaine manière emportée par son propre travail. » (De la grammatologie, Minuit, p. 39) Nous dirons qu'elle l'est d'autant plus chez Merleau-Ponty dans la mesure où ce dernier ne pratique pas cette « subversion» 
de façon tout à fait consciente, et qu'il faudra la distance critique de Le visible et l'invisible pour en apprécier (justement ou pas) les principales tensions.

Par ailleurs, les affiliations que Rainville retrace entre la pensée de Heidegger et celle de Merleau-Ponty au niveau des notions d'être au monde et de transcendance ne sont-elles pas un indice supplémentaire en faveur d'un dépassement d'une philosophie de la conscience, même si ce dépassement s'opère au sein de l'appareillage conceptuel de la métaphysique ?

Quoiquili en soit de la réponse à ces questions, l'essai de Rainville peut être considéré comme une contribution précieuse au débat entourant le “tournant » dans la pensée de Merleau-Ponty. On y retrouve une démarche rigoureuse alliée à un souci d'explorer de nouvelles possibilités; le tout débouche souvent - surtout dans la deuxième partie - sur des raisonnements saisissants qui, sans lever le voile sur les énigmes fondamentales, tiennent cependant compte de solutions possibles À cet égard, le chapitre 5 consacré à la question de la transcendance, est remarquable.

De quelque angle qu'on l'envisage, il me semble qu'il y a là une tentative d'amener l'expérience de pensée du philosophe à l'expression de son sens propre, mais sans chercher, comme le disait Merleau-Ponty lui-même dans ses derniers écrits, cette parole qui les terminerait toutes

Département de philosophie

Université de Montréal 